\title{
La salud en el marco de la psicología de la religión y la espiritualidad*
}

\section{The health in the mark of the psychology of the religion and the spiritualitY}

\author{
Japcy Margarita Quiceno** \\ Stefano Vinaccia
}

Universidad de San Buenaventura, Medellín, Colombia

Recibido: 24 de febrero de 2009

Revisado: 20 de abril de 2009

Aceptado: 5 de mayo de 2009

\section{Resumen}

El siguiente trabajo tiene como objetivo presentar una descripción del impacto que ha tenido en la salud "la psicología de la religión y la espiritualidad". Se muestra la evolución histórica de este constructo a lo largo del siglo XX, hasta llegar a las investigaciones basadas en la evidencia de las relaciones de la religión y la espiritualidad con la salud mental y física, y de investigaciones sobre los procesos de intervención en salud, enfocadas desde la espiritualidad. En conclusión, son muchas las investigaciones que plantean que la religión y la espiritualidad son variables moderadoras y amortiguadoras ante eventos traumáticos de la vida como lo es una enfermedad.

Palabras clave: espiritualidad, creencias religiosas, afrontamiento religioso, enfermedad, intervención en salud.

Artículo en investigación

Correspondencia: Japcy Margarita Quiceno. E-mail: japcyps@hotmail.com. Stefano Vinaccia. E-mail: vinalpi47@hotmail.com. Universidad San Buenaventura, Medellí, Colombia. Dirección Postal: A.A. 81240 Envigado, Antioquia. 


\section{Abstract}

The present work has as aim present a review of the impact that has had in the health "the psychology of the religion and the spirituality". There are examined the historical precedents of this concept from its origins up to coming in the middle of the 20th century where it begins and one gives increasing summit in the researches based on the evidence of the relations of the religion and the spirituality in the mental and physical health, equally an approximation is done to the researches on the processes of intervention in health focused from the spirituality. In conclusion, there are great the researches that raise that the religion and the spirituality are moderating and buffering variables before traumatic events of the life like it is a disease.

Key words: spirituality, religious beliefs, religious coping, disease, intervention in health.

\section{Introducción}

A lo largo de la historia de la humanidad se ha tratado de dar explicaciones a las manifestaciones, tanto físicas como mentales, del comportamiento humano, a través de los fenómenos espirituales y actividades religiosas, y se ha apropiado el concepto alma para dar respuestas a éstos.

Alma en latín significa anima y traduce en griego psiché, que indica principio que otorga la vida o mariposa. También se le ha denominado fe, espíritu, conciencia, mente, y frente a ella han existido dos tipos de controversia: "si se trata de una sustancia material o espiritual" o si es "individual o universal".

Por otro lado, espiritualidad proviene del latín espíritu, que significa respiración, es decir, vitalidad, y en cuanto a la relación con alma significa la capacidad de trascendencia (Volker, 2006).

\section{Historia y conceptos de la psicología de la religión y la espiritualidad}

A principios del siglo XX, la psicología da un carácter científico a los fenómenos del comportamiento espiritual-religioso con lo cual surge la llamada psicología de la religión, que tuvo aportaciones de todas las corrientes psicológicas en su desarrollo, como el conductismo, el psicoanálisis, la psicología humanista y transpersonal, especialmente en Europa y Estados Unidos donde surge como un área de la psicología propiamente. La psicología de la religión es la rama de la psicología aplicada y de la ciencia de la religión, que abarca las manifestaciones psicológicas vinculadas a la práctica religiosa. El centro de estudio son las creencias, actividades y experiencias religiosas desde el punto de vista psicológico (Font, 1999).

Entre los primeros fundadores se considera a Friedrich Schleiermacher con su obra "Psychologi" (1862), y como primer gran clásico de la especialidad de la corriente del pragmatismo, al psicólogo y filósofo William James con su obra "La variedad de la experiencia religiosa" (1902). Entre sus aportes está la diferencia entre la religión como institución (grupos religiosos y su organización), la religión como práctica personal (vivir experiencias místicas, independientemente de la cultura) y la diferencia entre religiosidad sana y religiosidad enfermiza. Para William James, factores emotivo-cognitivo-conductuales operaban en las experiencias religiosas.

Otros precursores de la época que dieron los primeros aportes científicos a la psicología de la religión fueron Edwin Starbuck en el año 1899, Stanley Hall del periodo de 1904-1917 y George Coe del año 1900 (Yoffe, 2007). Entre los teóricos principales del siglo XX se encuentran Sigmund Freud, Carl Jung, Alfred Adler, Gordon Allport, Daniel Bastón, Erik H. Erikson, Erich Fromm, Abraham Maslow y Viktor Frankl. Todos ellos hacen énfasis en la existencia de un Dios, en las prácticas y experiencias religiosas y categorizan a la religión como buena o mala, como medio de crecimiento y motivación o de enajenación del hombre y la sociedad (Faller, 2001). 
Gordon Allport en su libro The Individual and His Religión en 1950, ilustra cómo la gente puede usar la religión de formas diferentes y hace una distinción entre la religión madura, en la que la persona es dinámica y de mente abierta, y la religión inmadura, en la que la persona es egoísta y generalmente representa los estereotipos negativos que tiene sobre la religión. Más adelante, esta diferencia se define como "religión intrínseca" (fe genuina, sentida, devota) y "religión extrínseca" (asistencia a la iglesia para obtener el estado social). Luego, Daniel Batson, en 1993, define otra orientación: "religión como medio", "religión como final" y "religión como búsqueda" (RiveraLedesma \& Montero-López, 2007).

Es, entonces, a mediados del siglo XX que comienzan a tener auge las investigaciones relacionadas con espiritualidad, religión y salud (González, 2004; Hill et al., 2000). Más concretamente, en las tres últimas décadas se han venido publicando investigaciones en revistas médicas y psicológicas, en las cuales Harold G. Koenig, Kenneth I. Pargament, Pamela G. Reed, David B. Larson y Jeffrey S. Levin, aparecen como algunos de los pioneros que abrieron una nueva etapa para la investigación científica de la religión y la espiritualidad en el área de la salud (Moreira-Almeida, Lotufo \& Koenig, 2006). Lo anterior posibilitó que se incorporara académicamente el estudio de la espiritualidad, con lo cual surge el término "Psicología de la Religión y la Espiritualidad", que hace referencia a las experiencias y formas de participación religiosa, creencias y prácticas espiritual-religiosa, el afrontamiento religioso, la conversión y la fe (Yoffe, 2007). Para Koenig, McCullough y Larson (2001) la espiritualidad es la búsqueda personal para entender las respuestas a las últimas preguntas sobre la vida, su significado, y la relación con lo sagrado o lo transcendente, que puede o no conducir al desarrollo de rituales religiosos y la formación de una comunidad, mientras que la religión es un sistema organizado de creencias, prácticas, rituales, y símbolos diseñados para facilitar la cercanía a lo sagrado o transcendente (Dios, un poder más alto, o la verdad o la realidad última). Según Hood (2003, citado en Riso, 2006, p. 229).
Los constructos religión y espiritualidad se diferencian en una serie de oposiciones básicas no tan reconciliables, donde la religión es vista como sustantiva, estática, institucional y objetiva (no tan "buena" ni tan recomendable) y la espiritualidad es evaluada como funcional, dinámica, personal, subjetiva, basada en la experiencia (más "buena" y recomendable).

Beit-Hallahmi y Argyle (1997) proponen la consideración de la religión como una actitud compuesta de tres dimensiones: una cognitiva (creencias religiosas), comportamental (comportamientos religiosos y rituales más o menos institucionalizados y convencionales) y afectiva (vínculos entre el hombre y la transcendencia).

Las creencias religiosas han sido hasta el momento un tema de interés y estudio en lo que concierne al mantenimiento y recuperación de la salud. Existen un sinnúmero de investigaciones que incluyen variados aspectos que toman desde la evolución positiva de síntomas y conductas, hasta las intervenciones de salud (González, 2004). Las creencias religiosas se refieren a creencias acerca de la fe, lo sobrenatural y lo sagrado o divino, que conlleva a la adoración de una deidad o deidades, además puede referirse a los valores y prácticas basados en modelos ideológicos de un líder espiritual. A diferencia de otros sistemas de creencias, las creencias religiosas tienden a ser codificadas y se refieren más a las ideas que a las prácticas (Font, 1999).

Por otro lado, Pargament (1997), un psicólogo que se basa en los planteamientos de Lazarus y Folkman (1984) sobre afrontamiento al estrés, comienza a desarrollar desde finales de los años ochenta -y que puntualiza en 1997- su modelo de "coping religioso", que consiste en "aquel tipo de afrontamiento donde se utilizan creencias y comportamientos religiosos para prevenir y/o aliviar las consecuencias negativas de sucesos de vida estresantes, tanto como para facilitar la resolución de problemas".

El modelo de Pargament (1997) hace referencia a tres estilos de afrontamiento religioso: 1. estilo autodirigido: en el cual las personas confían en sí 
mismas más que en Dios para resolver sus problemas; 2. estilo elusivo o evitativo: en el que la responsabilidad es dejada en manos de la divinidad, y 3. estilo colaborativo: en el que se establece una dinámica compartida entre el hombre y Dios en el proceso de afrontamiento.

Estos tres procesos están también asociados al nivel de competencia, fundamentalmente al sentido de control personal, la habilidad para la solución de problemas y la autoestima. Así, el estilo autodirigido estaría asociado a un alto sentido de competencia personal y alta autoestima, con tendencia a la autonomía. El estilo evitativo estaría asociado a un bajo sentido de competencia personal, autoestima y habilidad de solución de problemas, así como mayor intolerancia a las diferencias interpersonales; enfatiza la autoridad externa y constituye así un estilo pasivo. Finalmente, el estilo colaborativo estaría asociado a un elevado sentido de control personal y autoestima, y bajo sentido de control por las circunstancias o el azar (Muñoz, 2003; Muñoz \& Moreno, 2003).

En definitiva, las estrategias de afrontamiento religiosas pueden ser realizadas de forma individual (rezo/oración personal) como colectiva (rezar u orar en grupos, participación en sitios de adoración) que activan mecanismos de adaptación por una relación personal con Dios o poder más alto que les puede dar esperanza en tiempos de crisis (Koenig, 2002). Estas prácticas religiosas proveen al individuo el crecimiento espiritual continuo, apoyo psicológico, propósito en la vida e interacción social.

Igualmente, a finales de los ochenta la enfermera Pamela G. Reed desarrolla la teoría de la autotrascendencia como un recurso psicosocial y espiritual importante en el desarrollo madurativo de las personas. La autotrascendencia se refiere a la capacidad del individuo para ampliar las fronteras personales y orientarse hacia perspectivas, actividades, y objetivos más allá de sí mismo sin negarse el valor del ser en el contexto presente. La autotrascendencia es definida como una am- pliación de los límites autoconceptuales de forma multidimensional: (a) hacia dentro, a través de experiencias introspectivas; (b) hacia afuera, mediante el aumento de las relaciones con los demás; (c) temporalmente, por medio de la integración del pasado y el futuro en el presente (Reed, 1991); y (d) mediante la conexión con dimensiones que están más allá del mundo perceptible -un poder más alto-(Reed, 2003; Coward, 2007).

\section{Modelos de espiritualidad en salud}

Recientemente, Koenig (2008) planteó cuatro modelos sobre la relación de la salud mental y física con la religión y la espiritualidad o secularidad (laicidad):

\section{Versión tradicional-histórica de espiritualidad}

Se caracteriza por la profunda religiosidad, dedicación al servicio de la religión y los miembros de una comunidad y, la enseñanza de las tradiciones de la fe a través del testimonio de vida. En esta versión, la religión, la espiritualidad y la secularidad (laicidad) son recursos que pueden promover valores morales, conexiones con otros, tranquilidad, armonía, bienestar, esperanza, rasgos positivos de carácter y estados mentales positivos como el propósito y significado de la vida.

La versión tradicional-histórica de espiritualidad puede ser estudiada usando los métodos de investigación de las ciencias sociales y conductuales. La espiritualidad es aquí distinta de las formas más superficiales o menos devotas de religión y de la secularidad (laicidad). Las personas espirituales pueden ser identificadas a través de medidas de participación religiosa que luego son comparadas con aquellas que son menos religiosas y con individuos seculares (laicos). La espiritualidad en esta versión es entonces un constructo completamente separado de las medidas de salud mental o física (Figura 1). 


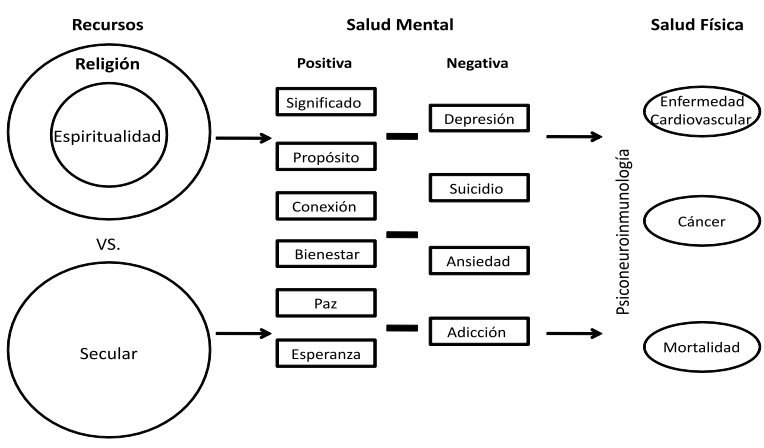

Figura 1. Versión tradicional-histórica de espiritualidad

\section{Versión moderna de espiritualidad}

Se caracteriza porque amplía o va más allá del constructo de religión tradicional. El término espiritualidad ha sido utilizado más ampliamente en la asistencia de salud, cuyo objetivo ha sido aplicarlo tanto a personas de diversos credos religiosos como aquellas que no lo tienen. Esto abre entonces una nueva categoría de "personas espirituales, pero no religiosas". Esta versión conceptualiza la comparación de la salud mental y física de los que son "espirituales religiosos", los que son "espirituales, pero no religiosos", y los que son "completamente seculares" (Figura 2).

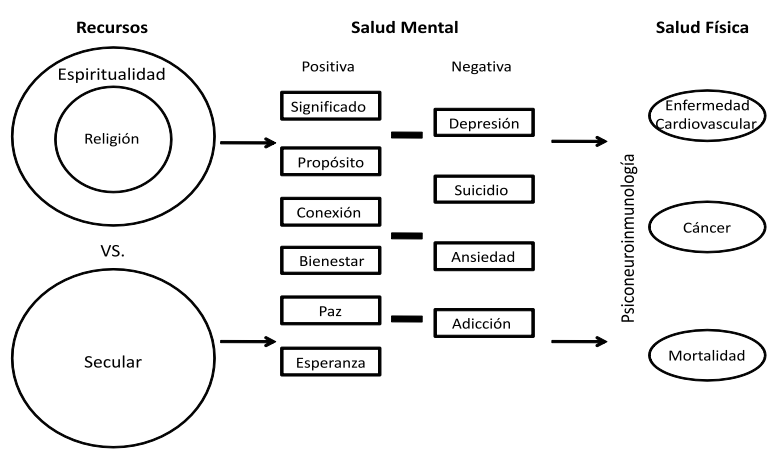

Figura 2. Versión moderna de espiritualidad

\section{Versión tautológica moderna de espiritualidad}

Aunque es similar a la anterior (versión moderna) se caracteriza porque se extiende hacia fuera incluyendo en su definición la salud mental positiva y los valores humanos. Este concepto de espiritualidad incluye no sólo indicadores religiosos tradicionales o una búsqueda de lo sagrado, sino también estados psicológicos positivos como propósito y significado de la vida, la conexión con los demás (la calidad de apoyo social), tranquilidad, armonía y bienestar. Se define como versión "tautológica" porque incluye indicadores de salud mental en la definición de espiritualidad, lo que asegura una correlación positiva entre estas dos variables (Figura 3).

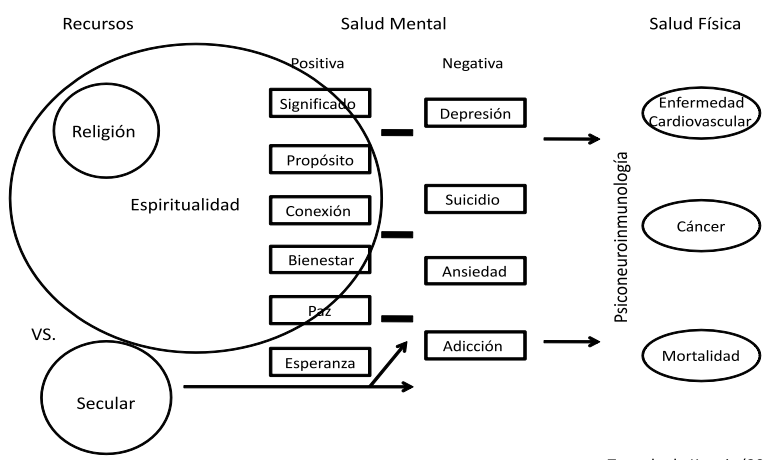

Figura 3. Versión tautológica moderna de espiritualidad

\section{Versión clínica moderna de espiritualidad}

Esta versión se caracteriza porque no sólo incluye las anteriores, es decir, el constructo de religión y los indicadores positivos de salud mental, sino también lo secular (laicismo) como elementos de su definición. En este modelo es considerado espiritual incluso lo agnóstico y lo ateo (Figura 4).

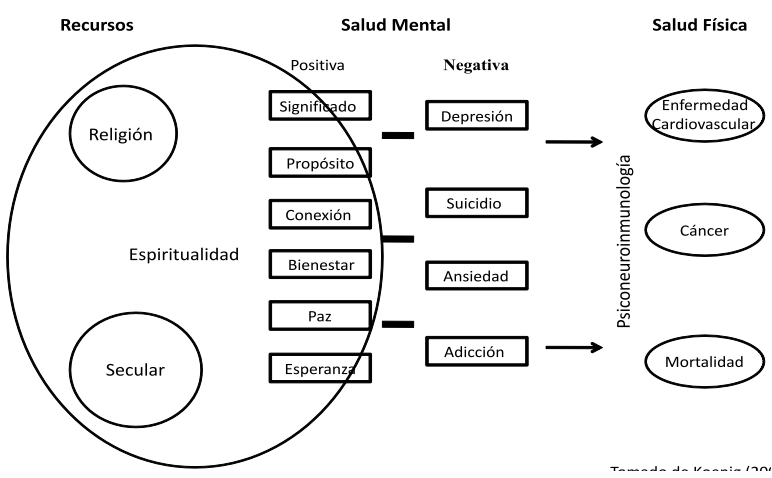

Figura 4. Versión clínica moderna de espiritualidad 


\section{Instrumentos de medida de orientación religiosa-espiritual en salud}

Ahora bien, a la par con el desarrollo teórico de la psicología de la religión y la espiritualidad, en el campo de la salud en los últimos 40 años se han venido desarrollando, diseñando y validando instrumentos psicométricos en diferentes versiones e idiomas para tener una medida objetiva de las creencias, prácticas y el afrontamiento espiritualreligioso, tanto en personas sanas como con alguna patología física o mental. En la Tabla 1 se presentan en orden cronológico algunos de los instrumentos más utilizados en investigación en salud para medir creencias y afrontamiento espiritual religioso, tanto en idioma inglés como en español.

Tabla 1. Principales pruebas generales y específicas para medir creencias y afrontamiento espiritual religioso en salud

\begin{tabular}{ll}
\hline \multicolumn{1}{c}{ Instrumentos } & \multicolumn{1}{c}{ Qué mide } \\
\hline $\begin{array}{ll}\text { Religious Orientation Inventory/ROI desarrollada origi- } \\
\text { nalmente por Allport y Ross (1967) }\end{array}$ & $\begin{array}{l}\text { Eváa separadamente la orientación intrínseca y extrínseca a la reli- } \\
\text { gión }\end{array}$
\end{tabular}

The Spiritual Well-Being Scale (SWBS) desarrollado ori- Esta escala proporciona un indicador general del bienestar espiritual. ginalmente por Paloutzian y Ellison (1982). Se cuenta Mide la percepción de calidad de vida espiritual, el bienestar religioso con una adaptación para México (Sub-escala de Rela- (que hace referencia a la autoevaluación de la relación de la persona ción con Dios de la Escala de Bienestar Espiritual) desa- con Dios) y el bienestar existencial (que hace referencia a la autoevarrollada por Montero-López y Sierra (1996) luación del sentido del propósito y la satisfacción con la vida)

Spiritual Perspective Scale (SPS) desarrollada originalmente por Reed (1986, 1987a)

Self-Transcendence Scale (STS) desarrollada originalmente por Reed (1987b)

Intrinsic/extrinsic measurement: I/E-revised desarro llada por Gorsuch y McPherson (1989)

Index of Core Spiritual Experiences/ INSPIRIT, desarro llado por Kass, Friedman, Laserman, Zuttermeister y Benson (1991)

Religious Coping Index ( $\mathrm{RCl}$ ) desarrollada originalmente por Koenig et al. (1992).

Spiritual Health Inventory (SHI), desarrollada originalmente por Highfield (1992)

Duke University Religious Index scale (DUREL) desarrollada por Koenig, Meador y Parkerson (1997)

The Spiritual Involvement and Beliefs Scale (SIBS), desarrollada originalmente por Hatch, Burg, Naberhaus y Hellmich (1997)

Systems of Beliefs Inventory/SBI desarrollado originalmente por Holland et al. (1998). Se cuenta con la versión para México (Inventario de Sistemas de Creencias/ ISC) desarrollada por Almanza, Monroy, Bimbela, Payne y Holland (2000)
Esta escala hace referencia al conocimiento de sí mismo, a un sentido de conexión con un ser de naturaleza superior o a la existencia de un propósito supremo

Mide la capacidad de la persona de buscar un sentido del bienestar a través de aspectos cognoscitivos, creativos, sociales, espirituales e introspectivos

Evalúa la religiosidad intrínseca y extrínseca. En la religiosidad intrínseca se miden creencias, actitudes y fe religiosa, y en la religiosidad extrínseca se evalúan relaciones interpersonales y sociales

Mide las experiencias cruciales que ha vivido una persona en relación con la existencia de un ser supremo

$\mathrm{El} \mathrm{RCl}$ es un cuestionario con tres ítems, diseñado para personas con enfermedades físicas y mentales. Mide el grado en el cual los participantes confían en sus actividades y creencias religiosas y cómo éstas le ayudan a afrontar su situación

Esta escala fue diseñada para pacientes oncológicos y comprende dos versiones: la Patient self-report SHI, que evalúa los sentimientos y conceptos de los pacientes relacionados con la salud espiritual, y la Nurse assessment $\mathrm{SHI}$, en el que las enfermeras reportan sus observaciones sobre los sentimientos y conceptos de los pacientes, relacionados con la salud espiritual

Es una escala de cinco ítems que mide la participación religiosa, en vez de la espiritualidad. Aunque es una escala originalmente diseñada para pacientes oncológicos, se puede aplicar en personas con otro tipo de enfermedades

Esta escala facilita el estudio científico del rol o la condición de la espiritualidad en la vida del paciente en relación con la atención médica

Mide las prácticas y creencias religiosas y espirituales al igual que el soporte social derivado de ellas. Aunque es una escala originalmente diseñada para pacientes oncológicos se puede aplicar a personas con otro tipo de enfermedades 
RCOPE desarrollado originalmente por Koenig, Pargament y Nielsen (1998) y Pargament, Koenig y Perez (2000). De esta escala se cuenta con una versión breve el Brief-RCOPE desarrollado originalmente por Pargament, Smith, Koenig y Perez (1998). Se cuenta además con una adaptación para México (Escala de Patrones positivos y negativos de métodos de afrontamiento religioso, Brief-RCOPE) desarrollada por Rivera-Ledesma y Montero-López (2005)

Escala de Afrontamiento Religioso ante la Soledad (ARS) desarrollada originalmente por Montero-López (1999)

Multidimensional Measure of Religiousness/Spirituality, Este instrumentos de 38 ítems estudia cuánto afectan o no a la salud los desarrollado por Fetzer Institute (1999). Se cuenta con siguientes factores: las experiencias espirituales diarias, el significado, una versión para adolescentes: el Brief Multidimensio- los valores, las creencias, el perdón, las prácticas religiosas privadas, el

nal Measure of Religiousness/Spirituality desarrolla- afrontamiento espiritual-religioso, el apoyo religioso, la historia espirida por Harris, Sherritt, Holder, Kulig, Shrier y Knight (2008)

Functional Assessment of Chronic Illness Therapy - Spiritual Well-Being (FACIT-Sp), desarrollada originalmente por Brady, Peterman, Fitchett, Mo y Cella (1999) y Peterman, Fitchett, Brady, Hernandez y Cella (2002)

Daily spiritual experiences long form (DES) desarrollado por Underwood y Teresi (2002). También se cuenta con una versión breve

Spiritual Coping Strategies (SCS), desarrollada originalmente por Baldacchino y Buhagiar (2003).

The SpREUK questionnaire, desarrollado originalmente por Ostermann, Bussing y Matthiessen (2004).

The Spirituality Index of Well-Being, desarrollado originalmente por Daaleman y Frey (2004)

The Spirituality Scale (SS), desarrollado originalmente por Delaney (2005)
Esta escala ha sido utilizada originalmente con personas de la tercera edad y sus seis ítems evalúan el conjunto de estrategias empleadas por un sujeto en el afrontamiento de sus sentimientos de soledad, caracterizadas por un centramiento en sus creencias y prácticas religiosas y espirituales tual-religiosa, el compromiso, la organización religiosa y la preferencia religiosa

Esta escala evalúa la religiosidad tradicional (factor de fe) y la espiritualidad (factor de significado y paz), este último factor de significado y paz tiene asociaciones con la adaptación psicológica. Aunque es una escala originalmente diseñada para pacientes oncológicos se puede aplicar en personas con otro tipo de enfermedades

Esta escala evalúa experiencias diarias de la vida espiritual en pacientes con enfermedades físicas

Esta escala comprende dos dimensiones que evalúan las estrategias de afrontamiento religioso y las estrategias de afrontamiento no religioso. Fue desarrollada originalmente con enfermos cardiacos, aunque se puede utilizar con población en general o que tenga otro tipo de enfermedades

Esta escala está compuesta por dos dimensiones: 1. Confianza en un poder más alto (religiosidad intrínseca); y 2. La Interpretación positiva de la enfermedad (se plantea que la enfermedad es una posibilidad de cambio y la curación es un proceso de acción para reflexionar sobre la vida)

Esta escala mide el bienestar psicológico espiritual en pacientes con enfermedades físicas y puede emplearse en estudios que evalúen calidad de vida. Comprende dos dimensiones, una que mide autoeficacia y la otra que evalúa esquemas de vida

El SS es un instrumento holístico que busca medir las creencias, intuiciones, opciones de modo de vida, las prácticas y los rituales representativos de la dimensión espiritual humana. Ha sido diseñado, además, para evaluar el efecto de programas de intervención espiritual

\section{Espiritualidad y salud mental y física}

El concepto de espiritualidad ha ido cobrando cada vez más importancia, sobrepasa los límites de la religión y de la moral, especialmente en el mundo occidental, hasta convertirse en un constructo estudiado ampliamente por el campo de la salud (Koenig, 2008). Actualmente, se cuenta con más de 1.200 estudios sobre espiritualidad y religión en el área de la salud, y es en los últimos veinte años cuando más se ha encontrado una fuerte relación entre religiosidad-espiritualidad y salud física y mental en todo tipo de enfermedad. Incluso se ha abordado en procesos de divorcio y crisis emocionales (Koenig et al., 2001; Baldacchino \& Buhagiar, 2003; Moreira-Almeida et al., 2006).

En cuanto a la salud física los estudios han estado centrados particularmente en la actividad inmunológica, las neoplasias y enfermedades cardiovas- 
culares, cerebrovasculares, neurológicas y dolor (Koenig et al., 2001; Guimarães \& Avezum, 2007; Peres, Arantes, Lessa \& Caous, 2007), y en cuanto a la salud mental los estudios han estado enfocados en las adicciones, el suicidio, la delincuencia, la ansiedad-depresión, el estrés, la esquizofrenia, el psicoticismo y los trastornos bipolares (Koenig et al., 2001; Mueller, Plevak \& Rummans, 2001; Moreira-Almeida et al., 2006; Dalgalarrondo, 2007; Koenig, 2007a). En general, en todos estos estudios se han encontrado relaciones positivas y causales (Guimarães \& Avezum, 2007).

Las investigaciones basadas en la evidencia plantean que aunque las personas no sean sumamente religiosas en su diario vivir, pueden serlo en momentos de enfermedad, debido a la experiencia de pérdida de control personal, frente a la situación que los lleva a la búsqueda de un poder más alto o un Dios para encontrar los propósitos de la vida y enfrentar situaciones estresantes (Koenig, George \& Siegler, 1988).

Es así como las estrategias de afrontamiento religiosas y existenciales pueden ayudar a la gente a afrontar las enfermedades crónicas en el tiempo $y$, consecuentemente, el afrontamiento espiritual-religioso puede mejorar la calidad de vida, brindar bienestar psicológico, felicidad, emociones positivas, disminuir los niveles de ansiedad, depresión y los comportamientos adictivos y suicidas, como ha sido planteado en algunos estudios en los que se ha evaluado la influencia positiva del afrontamiento espiritual-religioso en enfermedades como VIH, neoplasias, Enfermedad Pulmonar Obstructiva Crónica (EPOC) e, incluso, el estrés postraumático causado por terrorismo (MoreiraAlmeida et al., 2006; Koenig, 2007b; Panzini, Da Rocha, Bandeira \& Fleck, 2007; Peres, MoreiraAlmeida, Nasello \& Koenig, 2007; Meisenhelder \& Marcum, 2009).

Por otro lado, la literatura plantea que el afrontamiento espiritual como la oración, la contemplación, el yoga, qi gong, el tai chi, el budismo zen, la meditación trascendental, la relajación, la visualización, escuchar música y hacer contacto con la naturaleza, entre otras técnicas, tienen un impacto favorable en los procesos cerebrales, en la actividad eléctrica de los neurotransmisores, en el sistema nervioso, en el sistema límbico, en el sistema serotoninérgico y en las respuestas del organismo, pues se observa la presencia de menos niveles de cortisol plasmático y mejor funcionamiento del sistema inmune, se produce disminución del metabolismo en general, menor consumo de oxígeno y bióxido de carbono, disminución de la frecuencia del ritmo cardiaco, aumento de las ondas cerebrales lentas, disminución de los niveles de hipertensión arterial y estado de bienestar en general (Navas \& Villegas, 2006; Navas, Villegas, Hurtado \& Zapata, 2006). La relación mentecuerpo que posibilitan estas técnicas ayuda en última instancia al autoconocimiento, a una mejor adhesión a los tratamientos, mejores estilos de vida saludables y una favorable calidad de vida en general (Moreira-Almeida et al., 2006).

\section{Estudios empíricos sobre intervención espiritual en salud}

Muchos estudios epidemiológicos indican que espiritualidad y religión están asociadas con las medidas de la salud, y aunque no hay muchos estudios en el área de intervención espiritual, se observa que se está dando un aumento paulatino y creciente sobre el diseño de intervenciones que hagan referencia al valor de la espiritualidad en la salud (Kennedy, Abbott \& Rosenberg, 2002; Cunningham, 2005; Martinez, Smith \& Barlow, 2007).

Esto se ha expuesto reiteradamente incluso desde el siglo pasado por Hawks, Hull, Thalman y Richins (1995) quienes desarrollaron una revisión de literatura que abarcó los años 1964 a 1994 y cuyo objetivo fue examinar las definiciones sobre la salud espiritual; proveer una visión general de algunos métodos de intervención eficaces que pueden aumentar la salud espiritual; y dar una idea general de las relaciones potenciales entre las intervenciones de salud espiritual y los resultados de salud en los niveles conductual, emocional y físico.

Los autores encontraron que las técnicas de imaginería, la meditación y las actividades de apoyo social pueden servir para trabajar elementos relacionados con la salud espiritual como el signifi- 
cado y el propósito en la vida, el autoconocimiento y el conectarse consigo mismo, con los demás y con un poder más alto. En la revisión también fueron relevantes los cambios positivos hallados sobre las conductas de salud como la comunicación, la adhesión al tratamiento y el cumplimento de la dieta. Además, se encontró una gran variedad de resultados benéficos en la salud física y emocional como la reducción de enfermedades cardiacas y de mortalidad por cáncer, y la disminución de la ansiedad y de los estados de ánimo negativos.

En Estados Unidos, Yanek, Becker, Moy, Gittelsohn y Koffman (2001) desarrollaron un estudio cuyo objetivo fue evaluar el impacto sobre los perfiles de riesgo cardiovasculares de mujeres afroamericanas mayores de 40 años, después de un año de participación en un programa basado en nutrición y estrategias de actividad física. Hubo tres grupos, el primero, de intervención estándar de grupo conductual $(n=188)$, un segundo, de intervención estándar complementado con las estrategias espirituales $(n=267)$, y un tercer grupo, de estrategias de autoayuda ( $n=74) .529$ mujeres de 16 iglesias fueron evaluadas en una línea de base, antes y después de un año de la intervención, y las intervenciones fueron de 20 sesiones.

La intervención de comportamiento estándar consistió en educación sobre nutrición y actividad física (de acuerdo con las capacidades de las asistentes) con aeróbicos, caminar enérgicamente, ejercicios en el agua y ejercicios de boxeo.

La Intervención estándar con componentes de estrategias espirituales consistió en que las iglesias que ofrecieron la intervención espiritual recibieron las mismas sesiones que las iglesias estándar de intervención, con la adición de componentes espirituales contextualizados de acuerdo con cada iglesia, allí las sesiones se basaron en la oración grupal y mensajes sobre salud, guiados por los textos religiosos, y las actividades físicas incluyeron aeróbicos con música espiritual o evangélica (GodSpell, que en castellano se traduce como "llamada de Dios").
Hubo, además, soporte social religioso en el que el líder hace llamadas telefónicas a los participantes para motivarlos a que asistan, también hubo boletines en las iglesias que incluyeron recordatorios de las sesiones semanales que llevaban impresos mensajes positivos, como: comer sanamente, desarrollar actividades físicas y estar alegres. Estos mensajes estaban, a su vez, acompañados por pasajes de las Escrituras.

La intervención de autoayuda fue incluida como un plan de contingencia de referencia o de control que implicó el empleo de lecturas de folletos de la Asociación Americana del Corazón que hablaban sobre el comer sano y desarrollar actividades físicas. Los resultados indicaron que no hubo diferencias significativas entre el grupo de Intervención de comportamiento estándar y el grupo de Intervención estándar con componentes de estrategias espirituales, lo que llevó a unificarlos en un solo grupo llamado Intervención estándarespiritual y compararlos con el grupo de Intervención de autoayuda.

En general, hubo mejoras significativas en el grupo de Intervención estándar-espiritual respecto a los niveles de peso corporal, la circunferencia de cintura, tensión arterial sistólica, niveles de calorías y grasas, y niveles de sodio. En el grupo de Autoayuda hubo una mejoría sólo en los niveles de grasas. En el grupo de Intervención estándarespiritual, las mujeres, en el transcurso de un año, tuvieron una pérdida de peso muy significativa. Los autores concluyeron que la Intervención estándar-espiritual favorece un importante cambio en los perfiles de riesgos cardiovasculares, lo que no se vio reflejado en el grupo de Autoayuda.

Kennedy et al. (2002) desarrollaron en Estados Unidos una investigación cuyo objetivo fue evaluar si la participación en un programa de dos días y medio de duración para pacientes cardíacos y sus parejas causaba cambios en la espiritualidad, y si estos cambios estaban relacionados con cambios en la vivencia de la cólera, en el significado de bienestar en la vida, y en la confianza en el manejo de sus problemas. Participaron 72 pacientes, quienes diligenciaron cuestionarios antes y después del programa, que incluyó análisis y 
discusión sobre estilos de vida saludables, alimentación, manejo del estrés, comunicación, apoyo social y principios básicos de sanación espiritual. Las prácticas empíricas incluían el yoga, la meditación, la visualización y la oración.

Los resultados indicaron que el $78 \%$ de los pacientes tuvieron un incremento en la espiritualidad después de la terminación del programa. Los cambios encontrados en la espiritualidad estuvieron positivamente asociados con un aumento significativo en la percepción del bienestar en la vida, la autoconfianza en el manejo de problemas y una tendencia en la disminución de enfado o enojo. Los autores concluyeron que muchos pacientes y sus familias consideran la espiritualidad como un elemento importante de la salud en sus vidas.

En Canadá, Cunningham (2005) describió un estudio exploratorio sobre los efectos de un curso breve psicoeducativo cuyo objetivo fue enfatizar en los aspectos espirituales del afrontamiento y la salud. Participaron 97 pacientes con diferentes tipos y etapas de neoplasias en ocho sesiones. Se hizo un pre-test - pos-test y un seguimiento a los seis meses de la finalización del estudio. Los resultados indicaron mejoras significativas inmediatamente después de la intervención (antes de los seis meses), sin embargo, estas mejoras se empezaron a percibir a partir de la mitad de la octava semana de tratamiento. En las tareas para la casa, diseñadas en el programa, los pacientes debían trabajar arduamente sobre asuntos relacionados con las "dudas sobre la existencia de un Dios, la justicia y el perdón, la culpabilidad, la proyección, el autoconocimiento, y el significado del amor".

Cuando el curso avanzó, muchos afirmaron poder aceptar de mejor forma su condición y experimentar una visión más positiva del significado de sus vidas, se integraron con una apreciación más sensible para los acontecimientos de vida diaria y tuvieron menos tendencia a estar en desacuerdo con los demás. Se concluyó que la visualización en la terapia de grupo en los pacientes con cáncer puede ser de gran beneficio cuando se aborda desde una perspectiva espiritual.
Otro estudio, realizado en los Estados Unidos por Bormann et al. (2006), buscó analizar la eficacia de una intervención psicoespiritual sobre el distrés psicológico (los pensamientos intrusivos, el estrés, la ansiedad, la ira y la depresión), la calidad de vida y la satisfacción existencial espiritual de personas infectadas con el VIH. La intervención consistía en la repetición de un mantra (una palabra o frase con asociaciones espirituales repetida en silencio durante todo el día). En este estudio fueron asignadas 93 personas a dos condiciones experimentales: 46 participaron en la intervención y 47 personas conformaron el grupo control.

Los resultados indicaron que el grupo que utilizó el mantra mejoró significativamente en comparación con el grupo control, hubo reducción en rasgos de ira e incremento y profundidad en la fe espiritual. La utilización del mantra tuvo asociaciones inversamente proporcionales con los pensamientos intrusivos de los pacientes y se asoció positivamente con la calidad de vida, la satisfacción existencial espiritual, el significado/paz, y la fe espiritual. En conclusión, según este estudio, el mantra permite disminuir los niveles de estrés psicológico y favorece la satisfacción existencial espiritual en personas con diagnóstico de $\mathrm{VIH}$.

Moritz et al. (2006) desarrollaron un estudio en Canadá cuyo objetivo fue valorar la eficacia de un programa de espiritualidad en casa, enfatizando en los problemas emocionales en pacientes con estrés. La muestra no aleatoria del estudio estuvo conformada por 165 personas, 56 participaron en el grupo de Espiritualidad, 54 participaron en el grupo de Meditación y 55 conformaron el grupo Control.

Se evaluó la calidad de vida a través del cuestionario MOS SF-36 y el estado de ánimo mediante el Profile of Mood States (POMS) que mide seis estados de ánimo como: tensión/ansiedad, depresión/ melancolía, odio/hostilidad/angustia, vigor/actividad, fatiga/inercia y confusión/desconcierto. Comparando los resultados con el grupo control, sin ningún tipo de intervención, se hizo un pretest y pos-test doce semanas después de terminar la intervención. 
El programa de Espiritualidad se llevó a cabo en ocho sesiones mediante el uso de audiocasete: las sesiones uno y dos consistieron en explicar los aspectos divinos del yo y como éstos inducen sentimientos de acercamiento a la divinidad. En la sesión tres se llevaron a cabo prácticas de respiración y meditación que ayudan a las personas a conectarse con lo divino. La sesión cuatro consistió en la focalización de la práctica del autoconocimiento que se relacionó con el uso de los cinco sentidos. La sesión cinco consistió en la explicación del camino de la espiritualidad, el cual empieza con la fe, luego sigue la creencia para obtener la sabiduría y finalmente el entendimiento. La sesión seis consistió en enfatizar en la idea de construir la gratitud y la conexión con los demás. La sesión siete hizo referencia a la idea del desapego y de no juzgar los resultados de nuestras acciones. La sesión ocho concluyó el programa y enfatizó en los cambios que ocurren cuando la espiritualidad toma la vida y, finalmente, se introduce la visualización sobre el entendimiento de la conexión con los otros y la divinidad.

El grupo de Meditación enfatizó en ocho sesiones de meditación y relajación con ayuda de audiocasetes, mientras el grupo control desarrolló actividades normales de su vida diaria sin ningún tipo de intervención. Los resultados demostraron que el grupo de Espiritualidad tuvo una mejoría en el estado de ánimo y en el índice sumario de salud mental del cuestionario MOS SF-36 respecto al grupo de Meditación y el grupo Control, y que el grupo de Meditación tuvo mejores resultados respecto al grupo Control.

En Australia, Jorna, Ball y Salmon (2006) desarrollaron un estudio con el propósito de valorar la eficacia de un programa para incrementar la actividad física y promover la salud mental y espiritual, basado en la integración mente-cuerpo y espíritu. Participaron en este estudio 49 mujeres que pertenecían a un grupo religioso. Treinta de ellas conformaban el grupo Control y diecinueve terminaron las ocho semanas que comprendía el programa. Se realizaron pre-test y pos-test.

Los resultados indicaron que el grupo Experimental desarrolló mayor actividad física que el grupo
Control y tenían mejor salud mental y espiritual. Se concluyó que la actividad física es un recurso importante para mejorar no sólo el estado físico, sino también que es un medio que promueve la salud mental y espiritual.

En Brasil, Elias, Giglio, Pimenta y El-Dash (2007) desarrollaron un programa de entrenamiento sobre intervención RIME que consistió en la integración de técnicas de relajación mental y visualización de imágenes mentales con los elementos que representan la espiritualidad y con base en los relatos de EQM (experiencia de casi-muerte) para profesionales de salud. Este programa tenía como objetivo resignificar el dolor espiritual de los pacientes terminales.

Los participantes del estudio fueron una enfermera, un médico, tres psicólogos y una terapeuta alternativa que atendían a once pacientes terminales internados en diferentes hospitales entre los 27 y los 76 años de edad. Se utilizó en este estudio un análisis de resultados cuanti-cuali. En general, en los resultados se encontraron en el análisis de la vivencia de los profesionales, cinco categorías y quince subcategorías. En el análisis de la naturaleza del dolor espiritual, se encontró como categorías más prevalentes los miedos a la muerte expresados por la negación y por la percepción del cuadro clínico.

En resumen, las etapas para el desarrollo del RIME son: identificación del dolor espiritual por medio de entrevista semi-estruturada; condensación de los elementos del dolor espiritual, descritos predominantemente por pensamientos racionales y lógicos en un patrón de imágenes simbólicas; orientación de las técnicas de relajación mental y de visualización de imágenes mentales integradas a los elementos que componen la naturaleza de la espiritualidad; y sesiones de orientación familiar como forma complementaria a la aplicación de la intervención terapéutica.

En la aplicación del RIME se observaron diferencias estadísticamente significativas $(p<0,0001)$ al final de las sesiones, en el que los enfermos relataron mayor nivel de bienestar en comparación con el 
inicio de las sesiones, lo cual fue medido a través de una escala EVA (Escala Visual Analógica).

Los autores concluyen que el programa de entrenamiento propuesto es eficaz para preparar profesionales de la salud para el uso de la intervención RIME, capacitándolos para el proceso de cuidar y prestar asistencia espiritual según una perspectiva académica. Los resultados además sugirieron que el RIME posibilita la resignificación del dolor espiritual en pacientes terminales.

Martínez et al. (2007) desarrollaron en Estados Unidos un estudio con el propósito de evaluar las opiniones que tienen las personas sobre las experiencias en la intervención religiosa en psicoterapia. Participaron en este estudio 152 personas de un Centro de Asesoramiento Religioso adscrito a una Universidad patrocinada por la iglesia Mormona. Las sesiones se realizaron tanto dentro como fuera de las intervenciones de psicoterapia. Se realizó una encuesta para determinar cuáles intervenciones eran más apropiadas en concepto de los participantes, si las que son dentro de las intervenciones o las que son realizadas por fuera de las sesiones.

Los resultados indicaron que las intervenciones fuera de sesiones eran más apropiadas que las sesiones internas, pero los participantes relataron que las sesiones internas contenían mayor ayuda. Mientras que en ambos tipos de intervención (dentro y fuera) los participantes reportaron como más apropiadas el empleo de referencias de pasajes de las escrituras, enseñanza de conceptos espirituales, intervenciones de perdón, participación religiosa con las necesidades de la comunidad y asesoría espiritual. Se concluye en este estudio que algunas intervenciones pueden ser o no efectivas en algunos casos.

También en los Estados Unidos, Delaney y Barrere (2008) llevaron a cabo un estudio con el objetivo de evaluar la influencia de una intervención en una muestra de 46 enfermos cardiacos sobre la psico-espiritualidad. La intervención consistió en el uso de técnicas de imaginería acompañada de música, que en un primer momento fue conducida por un terapeuta. Luego, tres veces a la se- mana, en un periodo de un mes, los pacientes lo practicaban en sus hogares mediante la dirección de un disco compacto (cd). Como conclusión de este estudio se sugiere que la música y las imágenes metafóricas pueden mejorar las condiciones de salud, y pueden reducir la ansiedad estadorasgo en personas con una enfermedad cardiaca.

\section{Conclusiones}

Es bien conocido que en los últimos treinta años existe un sinnúmero de investigaciones en el campo de la salud desde una perspectiva de la Psicología de la religión y la espiritualidad. Éstos han sido llevados a cabo principalmente por médicos psiquiatras, enfermeras y trabajadores sociales, con una menor presencia de psicólogos. Por lo anterior, se entiende que los hallazgos investigativos tengan mayor prevalencia de la relación de la religión y la espiritualidad sobre la salud mental en comparación con los de enfermedades crónicas u otras dolencias físicas (Moreira-Almeida et al., 2006).

La religión y la espiritualidad mediante sus prácticas de meditación y el uso de técnicas como la relajación y la imaginería, así como el soporte del grupo o social, principalmente, posibilitan, en última instancia, estados de "tranquilidad" que favorecen los procesos cognitivos y la salud mental y física en las personas, en tres aspectos: consigo mismo, con los demás y con el futuro, lo que implica que puedan ser empleadas como estrategias terapéuticas en procedimientos psicológicos multimodales.

Por otra parte, la mayoría de las investigaciones han sido desarrolladas y publicadas en países anglosajones, y es de anotar la escasa investigación empírica sobre el tema en América Latina, a pesar de la importancia histórica y cultural de la religión y la espiritualidad en las poblaciones hispanoparlantes. Sin embargo, se destacan los estudios desarrollados en México por Armando RiveraLedesma, María Montero-López Lena y José de Jesús Almanza Muñoz, y en Brasil por Alexander Moreira-Almeida y su grupo de investigación.

De acuerdo con los estudios revisados, la experiencia religiosa y existencial se hace más viven- 
cial y cobra significado cuando se está en momentos de crisis y cuando se percibe que el control sobre la vida es incierto.

La Psicología de la religión y la espiritualidad, cuando se enmarca en el estudio de enfermedades o patologías crónicas, se encontraría entonces en un punto de convergencia entre la psicología de la salud y la psicología positiva al considerar los procesos de salud-enfermedad desde un enfoque de promoción, prevención e intervención positivos (Yoffe, 2007; Carr, 2007). A lo anterior, podría denominársele entonces "Psicología de la religión y espiritualidad de la salud".

\section{Referencias}

Almanza, J., Monroy, M., Bimbela, A., Payne, D.K. \& Holland, J.C. (2000). Spanish Version of the Systems of Belief Inventory (SBI-15-R): Cross Cultural Research on Spiritual and Religious Beliefs. Psychosomatics, 41 (2), 158.

Allport, G.W. \& Ross, J.M (1967). Personal religious orientation and prejudice. Journal Personality Social Psychology, 5, 432-443.

Baldacchino, D.R. \& Buhagiar, A. (2003). Psychometric evaluation of the Spiritual Coping Strategies scale in English, Maltese, backtranslation and bilingual versions. Journal of Advanced Nursing, 42, 558-570.

Beit-Hallahmi, B. \& Argyle, M. (1997). The psychology of religious behaviour, belief and experience. London: Routledge.

Bormann, J. E., Gifford, A. L., Shively, M., Smith, T. L., Redwine, L., Kelly, A., Becker, S., Gershwin, M., Bone, P. \& Belding W. (2006). Effects of Spiritual Mantram Repetition on HIV Outcomes: A Randomized Controlled Trial. Journal of Behavioral Medicine, 29 (4), 359376.

Brady, M.J., Peterman, A.H., Fitchett, G., Mo, M. \& Cella, D. (1999). A case for including spirituality in quality of life measurement in oncology. Psychooncology, 8 (5), 417-428.
Carr, A. (2007). Psicología positiva: La ciencia de la felicidad. Barcelona: Paidós

Cunningham, A.J. (2005). Integrating spirituality into a group psychological therapy program for cancer patients. Integrative Cancer Therapies, 4 (2), 178-86.

Coward, D. (2007). Teoría de la autotrascendencia. En A. Marriner y M. Raile (Eds.). Modelos y teorías en enfermería (pp. 447-467). Madrid: Elsevier.

Daaleman, T.P. \& Frey, B.B. (2004). The Spirituality Index of Well-Being: A New Instrument for Health-Related Quality-of-Life Research. Annals of Family Medicine, 2 (5), 499-503.

Dalgalarrondo, P. (2007). Estudos sobre religião e saúde mental realizados no Brasil: histórico e perspectivas atuais. Revista de Psiquiatría Clínica, 34 (1), 25-33.

Delaney, C. (2005). The Spirituality Scale: holistic assessment of the human spiritual dimension. Journal Holístico Nursering, 23 (1), 145-167.

Delaney, C. \& Barrere, C. (2008). The Influence of a Spirituality-Based Intervention on Psychospiritual Outcomes in a Cardiac Population. Holistic Nursing Practice, 22 (4), 210-219.

Elias, A.C., Giglio, J., Pimenta, C.A. \& El-Dash, L. (2007). Programa de treinamento sobre a intervenção terapéutica "relaxamento, imagens mentais e espiritualidade" (RIME) para re-significar a dor espiritual de pacientes terminais. Revista de Psiquiatría Clínica, 34 (1), 60-72.

Faller, G. (2001). Psychology versus religion. Journal of Pastoral Counseling, 36, 21-34.

Fetzer Institute/National Institute on Aging Working Group. (1999). Multidimensional Measurement of Religiousness / Spirituality for Use in Health Research. Kalamazoo, Michigan: Fetzer Institute. 
Font, J. (1999). Religión, psicopatología y salud mental introducción a la psicología de las experiencias religiosas y de las creencias. Barcelona: Paidós.

González, T.L. (2004). Las creencias religiosas y su relación con el proceso salud-enfermedad. Revista Electrónica de Psicología Iztacala, 7 (2), 19-29. http://www.iztacala.unam.mx/ carreras/psicologia/psiclin/2004-2b/vol7no2art2.pdf).

Gorsuch, R.L. \& McPherson, S.E. (1989). Intrinsic/extrinsic measurement: I/E-revised and single-item scales. Journal for the Scientific Study of Religion, 28, 348-354.

Guimarães, H. \& Avezum, A. (2007). Impacto da espiritualidade na saúde física. Revista de Psiquiatría Clínica, 34 (1), 88-94.

Harris, S.K., Sherritt, L.R., Holder, D.W., Kulig, J., Shrier, L.A. \& Knight, J.R. (2008). Reliability and Validity of the Brief Multidimensional Measure of Religiousness/Spirituality Among Adolescents. Journal of Religion and Health, 47 (4), 438-457.

Hatch, R.L., Burg, M.A., Naberhaus, D.S. \& Hellmich, L.K. (1997). The Spiritual Involvement and Beliefs Scale. Development and testing of a new instrument. Journal of Family Practice, 46, 476-486.

Hawks, S.R., Hull, M.L., Thalman, R.L. \& Richins, P.M. (1995). Review of spiritual health: definition, role, and intervention strategies in health promotion. American Journal of Health Promotion, 9 (5), 371-378.

Highfield, M.F. (1992). Spiritual health of oncology patients. Cancer Nursing, 15, 1-8.

Hill, P.C., Pargament, K.I., Hood, R.W., McCullough, M.E., Swyers, J.P., Larson, D.B. \& Zinbauer, B. (2000). Conceptualizing religion and spirituality: Points of commonality, points of departure. Journal for the Theory of Social Behavior, 30, 52-77.
Holland, J.C., Kash, K.M., Passik, M.K., Gronert, M.K., Sison, A., Lederberg, M., Russak, S.M., Baider, L. \& Fox, B. (1998). A brief spiritual beliefs inventory for use in quality of life research in Life-Threatening Illness. Psychooncology, 7, 460-469.

Jorna, M., Ball, K. \& Salmon, J. (2006). Effects of a holistic health program on women's physical activity and mental and spiritual health. The Journal of Science and Medicine in Sport, 9 (5), 395-401.

Kass, J.D., Friedman, R., Laserman, J., Zuttermeister, P.C. \& Benson, H. (1991). Research note: Health outcomes and a new index of spiritual experience. Journal for the Science Study of Religion, 30 (2), 203-211.

Kennedy, J.E., Abbott, R.A. \& Rosenberg, B.S. (2002). Changes in spirituality and well-being in a retreat program for cardiac patients. Alternative Therapies in Health and Medicine, 8 (4), 64-73.

Koenig, H.G. (2002). Religion, congestive heart failure and chronic pulmonary disease. Journal of Religion and Health, 41, 263-278.

Koenig, H.G. (2007a). Religião, espiritualidade e transtornos psicóticos. Revista de Psiquitria Clínica, 34 (1), 95-104.

Koenig, H. G. (2007b). Religion and Remission of Depression in Medical Inpatients with Heart Failure/Pulmonary Disease. The Journal of Nervous and Mental Disease, 195 (5), 389-395.

Koenig, H.G. (2008). Concerns about Measuring "Spirituality" in Research. The Journal of Nervous and Mental Disease, 196 (5), 349-355.

Koenig, H.G., Cohen, H.J., Blazer, D.G., Pieper, C., Meador, K.G., Shelp, F., Goli, V. \& DiPasquale, B. (1992). Religious Coping and Depression Among Elderly, Hospitalized Medically Ill Men. American Journal of Psychiatry, 149, 1693-1700. 
Koenig, H.G., George, L.K. \& Siegler, I.C. (1988). The use of religión and other emotion-regulating coping strategies among older adults. The Gerentologist, 28, 303-310.

Koenig, H.G., McCullough, M. \& Larson, D.B. (2001). Handbook of religion and health: a century of research reviewed. New York: Oxford University Press.

Koenig, H.G., Meador, K.G. \& Parkerson, G. (1997). Religion index for psychiatric research: a 5 -item measure for use in health outcome studies. The American Journal of Psychiatry 154, 885-886.

Koenig, H.G., Pargament, K.I. \& Nielsen, J. (1998). Religious coping and mental health outcomes in medically ill hospitalized older adults. Journal of Nervous and Mental Diseases, 186, 513-521.

Lazarus, R.S. \& Folkman, S. (1984). Stress, appraisal and coping. Nueva York: Springer.

Martínez, J.S., Smith, T.B. \& Barlow, S.H. (2007). Spiritual Interventions in Psychotherapy: Evaluations by Highly Religious Clients. Journal of Clinical Psychology, 63 (10), 943-960.

Meisenhelder, J.B. \& Marcum, J.P. (2009). Terrorism, Post-traumatic Stress, Coping Strategies, and Spiritual Outcomes. Journal of Religion and Health, 48 (1), 46-57.

Montero-López, M. (1999). Inventario Multifacético de Soledad. Tesis Doctoral no publicada, Universidad Nacional Autónoma de México, México.

Montero-López, M. \& Sierra, C.L. (1996). Escala de Bienestar Espiritual: Un estudio de validación. La Psicología Social en México, AMEPSO, 6, 28-33.

Moreira-Almeida, A., Lotufo, F. \& Koenig, G.H. (2006). Religiousness and mental health: a review. Revista Brasileira de Psiquiatria, 28 (3), 242-50.
Moritz, S., Quan, H., Rickhi, B., Liu, M., Angel, M., Vintila, R., Sawa, R., Sorianao, J. \& Toews, J. (2006). A home study-based spirituality education program decreases emotional distress and increases quality of life-a randomized, controlled trial. Alternative Therapy Health Medicine, 12 (6), 26-35.

Mueller, P.S., Plevak, D.J. \& Rummans, T.A. (2001). Religious involvement, spirituality, and medicine: implications for clinical practice. Mayo Clinical Procedures, 76 (12), 1225-1235.

Muñoz, A. (2003). ¿Por qué las creencias religiosas funcionan como recurso de afrontamiento? El modelo de K.I. Pargament. Encuentros en Psicología Social, 1 (2), 280-283.

Muñoz, A. \& Moreno, M. (2003). Relevancia y variaciones en las estrategias de afrontamiento religioso-espirituales de jóvenes y adultos. Encuentros en Psicología Social, 1 (5), 273-277.

Navas, C. \& Villegas, H. (2006). Espiritualidad y salud. Revista Ciencias de la Educación, 1 (27), 29-45.

Navas, C., Villegas, H., Hurtado, R. \& Zapata, D. (2006). La conexión mente-cuerpo-espíritu y su efecto en la promoción de la salud en pacientes. Revista Venezolana de Oncología, 18 (1), 28-37.

Ostermann, T., Bussing, A. \& Matthiessen, P.F. (2004). Pilot study for the development of a questionnaire for the measuring of the patients' attitude towards spirituality and religiosity and their coping with disease (SpREUK). Forsch Komplementarmed Klass Naturheilkd, 11, 346-353.

Paloutzian, R.F. \& Ellison, C.W. (1982). Loneliness, spiritual well-being and the quality of life. In L.A. Peplau y D. Perlman (Eds.). Loneliness: A sourcebook of current theory, research and therapy (pp. 224-237). New York: John Wiley \& Sons. 
Panzini, R., Da Rocha, N., Bandeira, D. \& Fleck, M. (2007). Qualidade de vida e espiritualidade. Revista de Psiquiatria Clínica, 34 (1), 105-115.

Pargament, K.I. (1997). The Psychology of Religion and Coping: Theory, Research, Practice. New York: Guilford Press.

Pargament, K.I., Smith, B., Koenig, H.G. \& Perez, L. (1998). Patterns of positive and negative religious coping with major life stressors. Journal for the Scientific Study of Religion, 37, 710-724.

Pargament, K.I., Koenig, H.G. \& Perez, L.M. (2000). The many methods of religious coping: Development and initial validation of the RCOPE. Journal of Clinical Psychology, 56, 519-543.

Peres, M., Arantes, A.C., Lessa, P. \& Caous, C.A. (2007). A importância da integração da espiritualidade e da religiosidade no manejo da dor e dos cuidados paliativos. Revista de Psiquiatria Clínica, 34 (1), 82-87.

Peres, J., Moreira-Almeida, A., Nasello, A.G. \& Koenig, H.G. (2007). Spirituality and Resilience in Trauma Victims. Journal of Religion and Health, 46 (3), 343-350.

Peterman, A.H., Fitchett, G., Brady, M.J., Hernández, L. \& Cella, D. (2002). Measuring spiritual well-being in people with cancer: the functional assessment of chronic illness therapy--Spiritual Well-being Scale (FACIT-Sp). Annals of behavioral medicine, 24 (1), 49-58.

Reed, P.G. (1986). Religiousness among terminally ill and healthy adults. Research in Nursing and Health, 9, 35-41.

Reed, P.G. (1987a). Spirituality and well-being in terminally ill hospitalized adults. Research in Nursing and Health, 10, 335-344.

Reed, P.G. (1987b). Constructing a conceptual framework for psychosocial nursing. Journal of Psychosocial Nursing, 25 (2), 24-28.
Reed, P.G. (1991). Toward a nursing theory of self-transcendence: Deductive reformulation using developmental theories. Advances in Nursing Science, 13 (4), 64-77.

Reed, P.G. (2003). The theory of Self-Transcendence. In M.J. Smith y P. Liehr, (Eds.). Middle range theories in nursing. New York: Springer.

Riso, W. (2006). Terapia Cognitiva: Fundamentos teóricos y conceptualización del caso clínico. Bogotá, Colombia: Norma.

Rivera-Ledesma, A. \& Montero-López, M. (2005). Espiritualidad y religiosidad en adultos mayores mexicanos. Salud Mental, 28 (6), 51-58.

Rivera-Ledesma, A. \& Montero-López, M. (2007). Ejercicio clínico y espiritualidad. Anales de psicología, 23 (1), 125-136.

Underwood, L.G. \& Teresi, J.A. (2002). “The Daily Spiritual Experience Scale: Development, Theoretical Description, Reliability, Exploratory Factor Analysis, and Preliminary Construct Validity Using Health-Related Data". Annals of Behavioral Medicine, 24 (1), 22-33.

Volker, G. (2006). Filosofía de la Psicología. Barcelona: Herder.

Yanek, L.R., Becker, D.M., Moy, T.F., Gittelsohn, J. \& Koffman, D.M. (2001). Project Joy: faith based cardiovascular health promotion for African American women. Public Health Reports, $116(1), 68-81$.

Yoffe, L. (2007). Efectos positivos de la religión y la espiritualidad en el afrontamiento de duelos. Psicodebate, 7, 193-205. 